\title{
The Claustrum-Prefrontal Cortex Pathway Regulates Impulsive-Like Behavior
}

\author{
기ianfeng Liu, ${ }^{\star}$ Ruyan Wu, ${ }^{\star}$ Bernard Johnson, Jimmy Vu, Caroline Bass, and $\odot$ Jun-Xu Li \\ Department of Pharmacology and Toxicology, University at Buffalo, Buffalo, New York 14214
}

The claustrum connects with a broad range of cortical areas including the prefrontal cortex (PFC). However, the function of the claustrum (CLA) and its neural projections remains largely unknown. Here, we elucidated the role of the neural projections from the CLA to the PFC in regulating impulsivity in male rats. We first identified the CLA-PFC pathway by retrograde tracer and virus expression. By using immunofluorescent staining of the c-Fos-positive neurons, we showed that chemogenetic activation and inhibition of the CLA-PFC pathway reduced and increased overall activity of the PFC, respectively. In the 5-choice serial reaction time task (5-CSRTT), we found that chemogenetic activation and inhibition of the CLA-PFC pathway increased and reduced the impulsive-like behavior (i.e., premature responses), respectively. Furthermore, chemogenetic inhibition of the CLA-PFC pathway prevented methamphetamine-induced impulsivity, without affecting methamphetamine-induced hyperactivity. In contrast to the role of CLA-PFC pathway in selectively regulating impulsivity, activation of the claustrum disrupted attention in the 5-CSRTT. These results indicate that the CLA-PFC pathway is essential for impulsivity. This study may shed light on the understanding of impulsivity-related disorders such as drug addiction.

Key words: 5-CSRTT; claustrum; impulsivity; prefrontal cortex; rat

\section{Significance Statement}

The claustrum is one of the most mysterious brain regions. Although extensive anatomical studies demonstrated that the claustrum connects with many cortical areas, the function of the neural projections between the claustrum and cortical areas remain largely unknown. Here, we showed that the neural projections from the claustrum to the prefrontal cortex regulates impulsivity by using the designer drugs (DREADDs)-based chemogenetic tools. Interestingly, the claustrum-prefrontal cortex pathway also regulates methamphetamine-induced impulsivity, suggesting a critical role of this neural pathway in regulating impulsivityrelated disorders such as drug addiction. Our results provided preclinical evidence that the claustrum-prefrontal cortex regulates impulsivity. The claustrum-prefrontal cortex pathway may be a novel target for the treatment of impulsivityrelated brain disorders.

\section{Introduction}

Impulsivity is generally defined as actions which are poorly conceived, prematurely expressed, unduly risky, or inappropriate to the situation and that often results in undesirable consequences (Dalley et al., 2011). A deficit in impulse control is a crucial symp-

\footnotetext{
Received May 3, 2019; revised Nov. 3, 2019; accepted Nov. 4, 2019.

Author contributions: J.L. and J.-X.L. designed research; J.L., R.W., B.J., and J.V. performed research; C.B. contributed unpublished reagents/analytic tools; J.L. analyzed data; J.-X.L. wrote the paper.

This work was supported by the National Institutes of Health National Institute on Drug Abuse (Grant R21DA040777). The content is solely the responsibility of the authors and does not necessarily represent the official views of the National Institutes of Health. We thank Dr. Ken Wakabayashi for suggestions on immunology, and Dr. Wade Sigurdson and the University at Buffalo Confocal Microscopy Core for assistance.

The authors declare no competing financial interests.

*J.L. and R.W. contributed equally to this work.

Correspondence should be addressed to Jianfeng Liu at jliu66@buffalo.edu or Jun-Xu Li at junxuli@buffalo.edu. https://doi.org/10.1523/JNEUROSCl.1005-19.2019

Copyright $\odot 2019$ the authors
}

tom in several psychiatric disorders, such as drug addiction, attention-deficit/hyperactivity disorder, and schizophrenia.

Impulse control requires appropriate function and coordination of several brain systems. For example, the prefrontal cortex (PFC)-striatal circuitry plays essential roles in regulating cognition, attention, and impulsivity (Miller, 2000; Kim and Lee, 2011).

Recent studies showed that the claustrum (CLA), a brain region next to the insular cortex, directly innervates the PFC (Goll et al., 2015; Jackson et al., 2018). It was hypothesized that the CLA might be a center of consciousness because of the highest connectivity and specific architecture of the CLA (Crick and Koch, 2005; Goll et al., 2015). Recent studies suggested that the CLA interacts with many cortical areas and plays critical roles in some fundamental functions of the brain (Kim et al., 2016; Brown et al., 2017). However, the neurobiological function of the CLA is largely understudied.

Previous studies have used several behavioral tasks to measure impulsivity in animals. The 5-choice serial-reaction time task 
(5-CSRTT) is the most widely used task evaluating attention and impulsive-like behavior (Bari et al., 2008). In particular, the 5-CRSTT has been used to study the underlying mechanism, including neuroanatomy and neurochemistry of impulsivity (Robbins, 2002; Dalley et al., 2011). For example, it was shown that pathological alterations in the PFC are linked to impaired inhibitory control in the 5-CSRTT (Bari and Robbins, 2013).

In this study, by using the 5-CSRTT in rats, we aimed to investigate the function of the CLA-PFC pathway in regulating attention and impulsivity. We confirmed the connectivity between the CLA and the PFC by using fluorescent tracer and viruses. We examined the effects of chemogenetic modulation of the CLA and the CLA-PFC pathway on the performance of 5-CSRTT. We also investigated the effects of chemogenetic modulation of the CLA-PFC pathway on methamphetamine-induced impulsivity.

\section{Materials and Methods}

Subject. Adult male Sprague-Dawley rats (initial weight 250-280 g; Envigo) were housed individually on a 12/12 h light/dark cycle (behavioral experiments were conducted during the light period) with ad libitum access to water and food except during experimental sessions. Rats were maintained and experimental procedures were approved by the Institutional Animal Care and Use Committee, University at Buffalo, the State University of New York, and with the 2011 Guide for the Care and Use of Laboratory Animals (Institute of Laboratory Animal Resources on Life Sciences, National Research Council, National Academy of Sciences, Washington DC).

Drugs. Drugs used in this study included clozapine- $N$-oxide (CNO; Research Triangle, 13626-103; requested from National Institute of Drug Abuse Drug Supply Program), FluoroGold (hydroxystilbamidine; cata$\log \# 80014$, Biotium), and methamphetamine (METH; National Institute of Drug Abuse). All drugs were dissolved in $0.9 \%$ physiological saline. Doses of CNO (intracranial: $1 \mathrm{~mm}, 0.5 \mu \mathrm{l} / \mathrm{side}$; i.p., $1 \mathrm{mg} / \mathrm{kg}$ ), Fluoro-Gold ( $3 \%$ in saline, $0.3 \mu \mathrm{l} / \mathrm{side})$, and METH $(0.3$ and $1 \mathrm{mg} / \mathrm{kg}$, i.p.) were based on previous studies (Siemian et al., 2017; Wakabayashi et al., 2019).

Adeno-associated virus. To modulate the activity of the CLA, we bilaterally injected adeno-associated virus (AAV) expressing the excitatory DREADD (hM3D) or inhibitory DREADD (hM4D) into the CLA. All viruses (AAV2/10-CMV-hM3D-mCherry, AAV2/10-CMV-hM4DmCherry, and AAV2/10-CMV-mCherry) used in the present study were provided and verified by Dr. Caroline Bass, University at Buffalo.

Stereotaxic surgeries and intracranial injections. These procedures were based on our previous studies (Liu et al., 2017; Yan et al., 2018). The rats (weighing 280-300 g when surgery began) were anesthetized with ketamine and xylazine (75 and $5 \mathrm{mg} / \mathrm{kg}$, respectively, i.p.). We bilaterally injected AAV2/10-CMV-hM3D-mCherry, AAV2/10-CMV-hM4DmCherry, or AAV2/10-CMV-mCherry into the CLA $(0.6 \mu \mathrm{l} /$ side $)$ with 5 $\mu \mathrm{l}$ Hamilton syringes (\#84851) connected with 33 gauge injectors (\#7762-06, Hamilton). To maximize the selectivity of viral expression in the CLA, we microinjected AAV viruses into two adjacent coordinates for the CLA (each $0.3 \mu \mathrm{l} /$ side/coordinate): angle, $4^{\circ}$; anterior/posterior (AP): $+1.0 \mathrm{~mm}$; medial/lateral (ML): $\pm 4.84 \mathrm{~mm}$; dorsal/ventral (DV): -6.08 $\mathrm{mm}$; and angle, $4^{\circ}$; AP: $+1.8 \mathrm{~mm}$; ML: $\pm 4.22 \mathrm{~mm}$; DV: $-5.92 \mathrm{~mm}$. The viruses were microinjected at a rate of $0.05 \mu \mathrm{l} / \mathrm{min}$, and the injection needles were kept in place for an additional $5 \mathrm{~min}$ to allow for diffusion. Guide cannulae (26 gauge; Plastics One) were bilaterally implanted $1 \mathrm{~mm}$ above the PFC (Liu et al., 2017): angle, $10^{\circ}$; AP: $+2.55 \mathrm{~mm}$; ML: \pm 1.6 $\mathrm{mm}$; DV: $-2.5 \mathrm{~mm}$. The cannulae were anchored to the skull with three to four stainless-steel screws and dental cement. A stainless-steel dummy was inserted into each cannula to keep it patent and to prevent infection. The rats were allowed to recover for at least 1 week after stereotaxic surgery. CNO ( $1 \mathrm{~mm}, 0.5 \mu \mathrm{l} / \mathrm{side}$ ) or saline was freshly prepared before the experiments and microinjected bilaterally over $1 \mathrm{~min}$ via microinjection needles ( 33 gauge; Plastics One) that were $1 \mathrm{~mm}$ longer than the cannulae. The injection needles were kept in place for an additional $1 \mathrm{~min}$ to allow for drug diffusion. The cannula placements were confirmed in $20-\mu$ m-thick sections using Nissl staining under light microscopy after all behavioral tests.

\section{Experimental design and statistical analysis}

5-CSRTT. Rats were trained under a 5-CSRTT procedure according to a published protocol and our previous study (Bari and Robbins, 2013; Xue et al., 2018). Sessions were conducted in standard chambers designed for the 5-CSRTT (Med Associates) measuring within sound-attenuating, ventilated enclosures. Session programs were controlled and data collected through a PC-interface setup and Med-PC IV software (Med Associates). Briefly, sessions began with illumination of the house light and food magazine and delivery of one food pellet ( $45 \mathrm{mg}$ dustless precision pellets; Bio Serv). Once the food pellet was collected, the intertrial interval (ITI) began and only the house light was illuminated. At the end of the ITI, one of the five response holes on the chamber wall opposing the food magazine was illuminated for a brief amount of time, the stimulus duration (SD). A correct response into this hole within the limited hold (LH) period turned off the target stimulus, turned on the food magazine light, and delivered one food pellet. Once the food pellet was collected, the next ITI began. The target stimulus varied pseudorandomly between trials. A response into the non-target hole was considered an incorrect response, and a failure to respond was considered an omission; each caused a $5 \mathrm{~s}$ timeout period in which all chamber lights were extinguished, followed by an initiation of the next ITI. Responses during the ITI before target stimulus presentation also caused a timeout and were considered as premature responses.

Sessions lasted for 100 total trials or $60 \mathrm{~min}$, whichever occurred first, and rats were trained $7 \mathrm{~d}$ per week. At the onset of training, the SD, LH, and ITI were 30,30 , and $5 \mathrm{~s}$, respectively. Over the course of training, the $\mathrm{SD}$ and LH decreased according to each rat's performance (Bari et al., 2008). Once the SD and LH reached 2.5 and 5 s, respectively, the $\mathrm{LH}$ remained constant for the rest of the experiment. The SD was further adjusted by $0.25 \mathrm{~s}$ increments until performance was maintained at a stable level of $>70 \%$ accuracy and $<30$ omissions for three consecutive sessions. The final SD was set at $1.0 \mathrm{~s}$. The ITI was set at $5 \mathrm{~s}$ in a regular test, whereas it was increased to $15 \mathrm{~s}$ in the challenge test. The challenge test lasted for 100 total trials or $2 \mathrm{~h}$, whichever occurred first. In the present study, all rats finished the challenge test in $2 \mathrm{~h}$. Rats were always placed in the testing chambers and allowed to habituate for $5 \mathrm{~min}$ before the start of the session, and $3 \mathrm{~d}$ of additional training (the performance achieved the stable level of $>70 \%$ accuracy and $<30$ omissions) were interspersed between tests. CNO ( $1 \mathrm{~mm}, 0.5 \mu \mathrm{l} /$ side $)$ or saline $(0.5 \mu \mathrm{l} /$ side) was microinjected into the PFC 5 min before tests. Intraperitoneal injections of CNO $(1 \mathrm{mg} / \mathrm{kg})$ or saline $(1 \mathrm{ml} / \mathrm{kg})$ was conducted $30 \mathrm{~min}$ before tests.

Locomotor activity test. Locomotor activity was recorded by an infrared motion-sensor system (AccuScan Instruments) fitted outside plastic cages $(40 \times 40 \times 30 \mathrm{~cm}$; Liu et al., 2018). The plastic cages contained a thin layer of corn cob bedding and were cleaned between each test session. The Fusion activity-monitoring system software monitors infrared beam breaks at a frequency of $0.01 \mathrm{~s}$. The interruption of any beam not interrupted during the previous sample was interpreted as an activity score. The Versa Max animal activity monitoring software monitors the distance the rats traveled in $30 \mathrm{~min}$. The locomotor activity of the rats was measured 3 weeks after expression of AAV2/10-CMV-hM3D-mCherry or AAV2/10-CMV-hM4D-mCherry. CNO ( $1 \mathrm{~mm}, 0.5 \mu \mathrm{l} / \mathrm{side}$ ) or saline $(0.5 \mu \mathrm{l} /$ side $)$ was microinjected into the PFC $5 \mathrm{~min}$ before the test session started.

METH-induced hyperactivity. The described locomotor chambers were used. Three weeks after viral injection (AAV2/2/10-CMV-hM4D$\mathrm{mCherry}$ ) into the CLA, rats were habituated in the chambers for $2 \mathrm{~d}(45$ $\mathrm{min} / \mathrm{d}$ ). In the test, rats were placed in the chambers for $25 \mathrm{~min}$ habituation followed by saline $(1 \mathrm{ml} / \mathrm{kg}$, i.p.) or METH ( $1 \mathrm{mg} / \mathrm{kg}$, i.p.) injections. The two tests (saline/METH) were separated by $1 \mathrm{~d}$ with a $45 \mathrm{~min}$ habituation. A total of $20 \mathrm{~min}$ movement was recorded after injections (Siemian et al., 2017).

Immunofluorescent staining. Immunofluorescent staining methods were similar as described in our previous study (Liu et al., 2018). Rats 


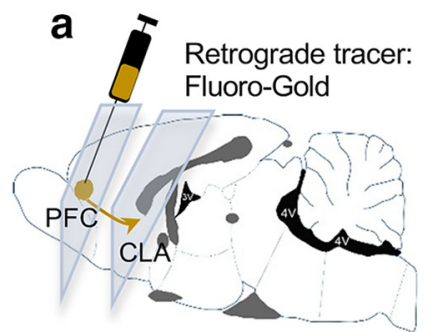

b Bregma $+3.0 \mathrm{~mm}$

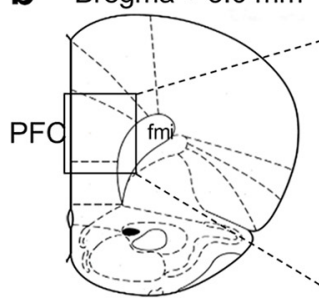

C Bregma

$+2.2 \mathrm{~mm}+1.6 \mathrm{~mm}$

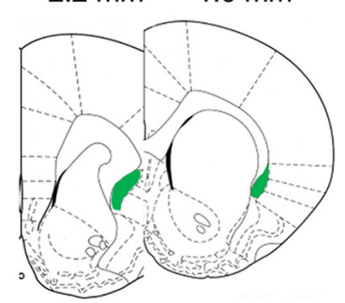

Fluoro-Gold/ DAPI

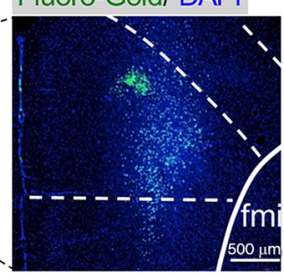

d
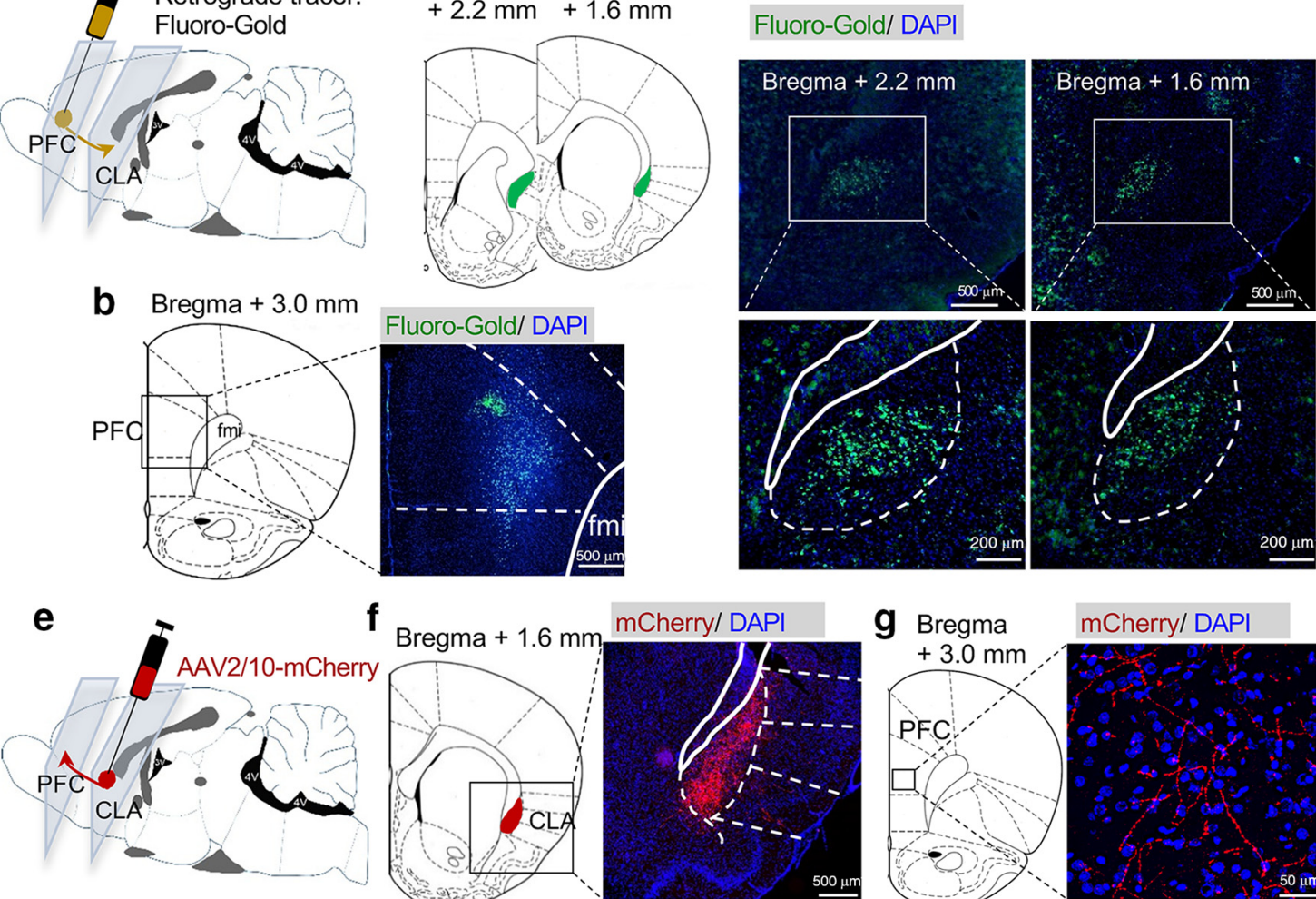

f

Bregma + $1.6 \mathrm{~mm}$
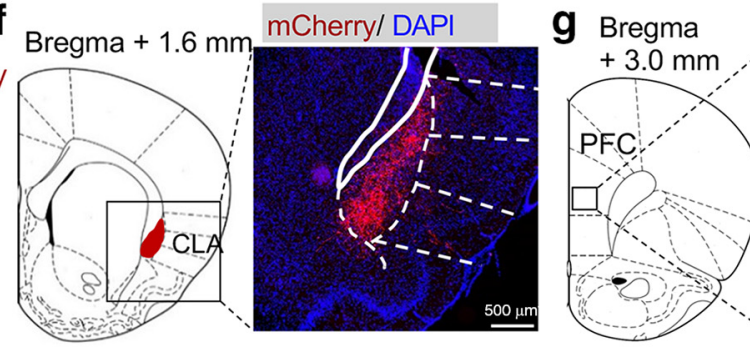

mCherry/ DAPI

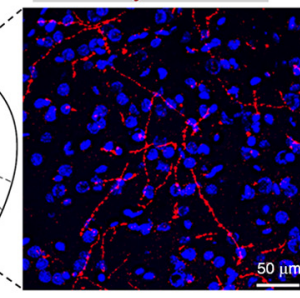

Figure 1. The claustrum sends neural projections to the prefrontal cortex. $\boldsymbol{a}$, Microinjection of retrograde beads FluoroGold into the PFC. $\boldsymbol{b}$, A representative image showing the FluoroGold in the PFC. $c$, Representative figures showing the location of the CLA. $\boldsymbol{d}$, FluoroGold microinjected into the PFC labeled cells in the CLA.e, Microinjection of AAV2/10-mCherry into the CLA.f, A representative figure showing expression of mCherry in the CLA. $g$, mCherry was detected in the neural terminals in the PFC after viral microinjection into the CLA.

were perfused with $4 \%$ paraformaldehyde, and the brains were removed and postfixed for $24 \mathrm{~h}$. For the c-Fos experiment, rats were perfused with $4 \%$ paraformaldehyde 90 min after microinjection of $\mathrm{CNO}$ or saline. The brains were then sectioned coronally with a microtome into 20 - $\mu$ m-thick sections. The free-floating tissue sections were washed three times $(10$ min each) in PBS followed by 50 min incubation in blocking buffer (3\% donkey serum, $0.3 \%$ TritonX100). Sections were then incubated overnight at $4^{\circ} \mathrm{C}$ in primary antibodies diluted in PBS with 3\% normal donkey serum and $0.3 \%$ Tween 20: anti-c-Fos (1:500), anti-mCherry (1:500), anti-FluoroGold (1:500). AlexaFluor 488-conjugated or AlexaFluor 594conjugated secondary antibodies were diluted 1:500 in PBS. All antibodies were purchased from Abcam. Sections were mounted with VECTASHIELD Hardset Antifade Mounting Medium with DAPI (cata$\log \# \mathrm{H}-1500$, Vector Laboratories) and stored at $4^{\circ} \mathrm{C}$. Images were taken with an Olympus IX51 fluorescence inverted microscope and Leica DMi8 fluorescence inverted microscope. For the c-Fos experiment, four to five sections representing the PFC of each animal were selected according to the brain atlas and microinjection sites. The number of c-Fospositive cells was counted manually and was averaged for each rat. All image acquisition and analyses were conducted by investigators blind to the experimental conditions.

Statistical analysis. All results were presented as mean \pm SEM, and were analyzed by GraphPad Prism 8 software. No statistical methods were used to predetermine sample sizes, but our sample sizes are similar to those reported in our previous studies (Xue et al., 2018). Behavioral tests were analyzed by ANOVAs (specifically stated in figures) followed by post hoc Bonferroni's test. The c-Fos results were analyzed by twotailed unpaired $t$ tests. $p<0.05$ was considered statistically significant. Injection sites and viral expression were confirmed for all animals. All rats showed correct placement of cannulae in the present study. Eight rats showing incorrect viral expression were excluded from the data analysis.

\section{Results}

The claustrum negatively regulates the activity of the PFC

To identify the neural projections from the CLA to the PFC, we first microinjected retrograde tracer FluoroGold retrocedes into the PFC in rats (Fig. 1a,b). We found that FluoroGold labeled neurons in the CLA but not neighboring brain areas (Fig. $1 c, d$ ). Then, we microinjected the adeno-associated virus (AAV)2/10mCherry into the CLA and examined the neural terminals in the PFC (Fig. 1e). Rats infused with AAV2/10-mCherry were perfused 3 weeks after virus infusion. The virus was microinjected into the CLA exclusively (Fig. $1 f$ ). We observed extensive terminals expressing mCherry in the PFC (Fig. 1g).

To modulate the activity of the CLA-PFC pathway, we used the designer receptors exclusively activated by DREADD-based chemogenetic tools (Roth, 2016). To selectively activate or inhibit the CLA-PFC pathway, we microinjected the AAVs expressing excitatory DREADD hM3Dq or inhibitory DREADD hM4Di into the CLA and microinjected CNO via the cannulae implanted into the PFC ( $1 \mathrm{~mm}, 0.5 \mu \mathrm{l} / \mathrm{side}$; Fig. $2 a-c)$. To determine the validity of our methods that we could modulate the activity of this pathway, we performed an immunofluorescence experiment to examine the expression of c-Fos, a marker of neuron activation, in the PFC (Fig. $2 d, e$ ). We found that microinjection of CNO into the PFC of rats expressing hM3Dq and hM4Di in the CLA suppressed and increased the number of overall c-Fos-positive neurons in the PFC, respectively (unpaired two-tailed $t$ test: hM3Dq-saline vs hM3Dq-CNO, $t_{(12)}=2.57, p=0.02$; hM4Disaline vs hM4Di-CNO, $t_{12}=5.45, p<0.001$; Fig. $\left.2 f\right)$. Together, 

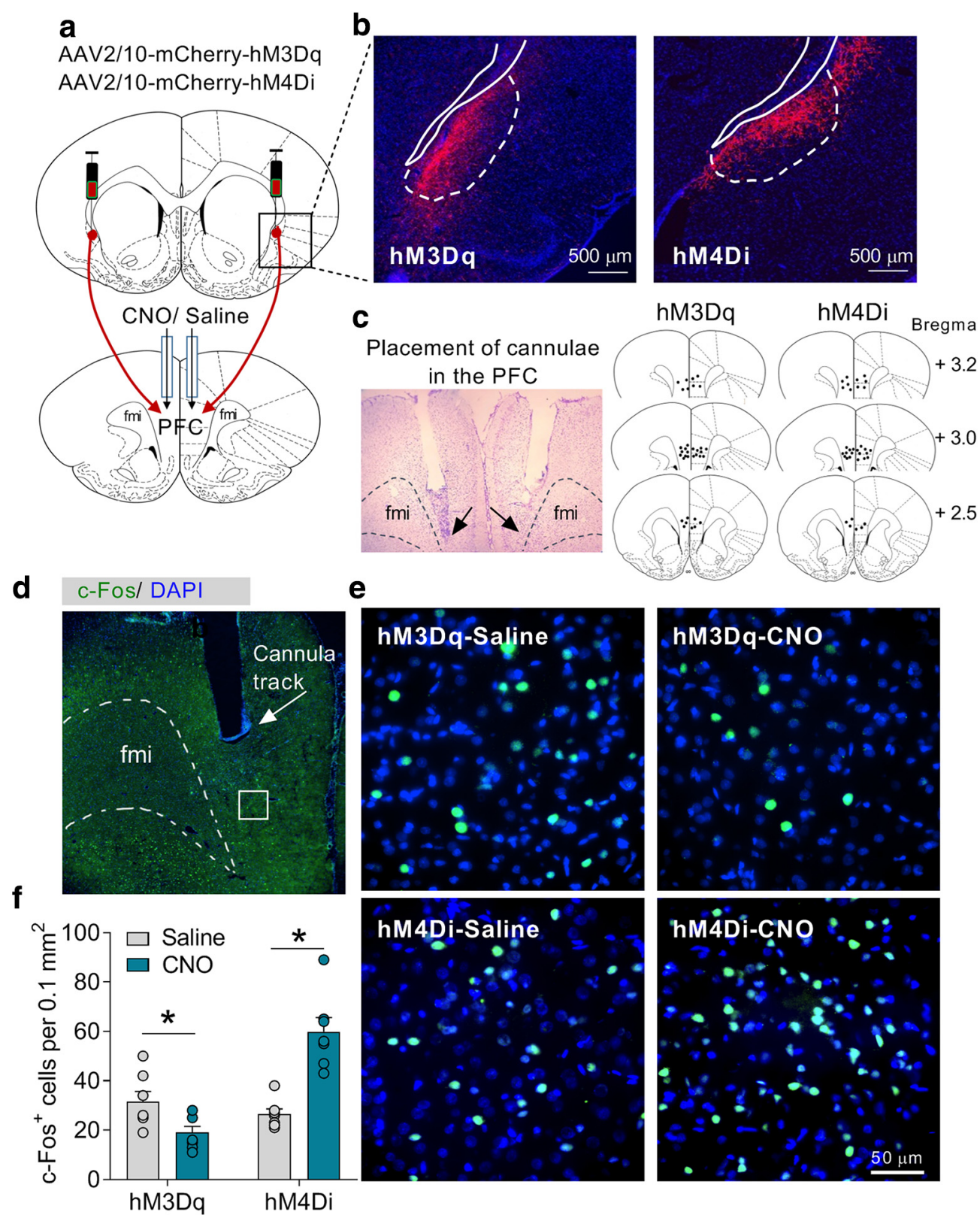

g

Locomotion
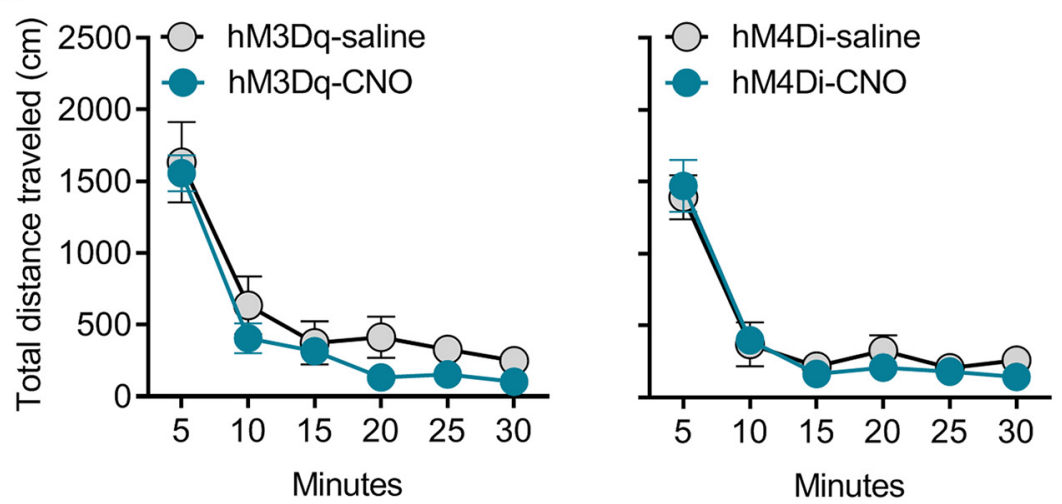

Figure 2. Chemogenetic modulation of the CLA-PFC pathway did not affect locomotion. $\boldsymbol{a}$, Representative figure showing chemogenetic modulation of the CLA-PFC pathway. $\boldsymbol{b}$, Representative figures showing expression of hM3Dq-mCherry and hM4Di-mCherry in the CLA. c, Brain atlas showing microinjection sites. $\boldsymbol{d}$, Representative figure showing cannulae placement and c-Fos expression in the PFC under low magnification ( $4 \times$ objective). $\boldsymbol{e}$, Representative figures of c-Fos expression of different groups of rats under high magnification (20× objective). $\boldsymbol{f}$, Chemogenetic activation and inhibition of the CLA-PFC pathway reduced and increased the number of c-Fos-positive cells in the PFC, respectively. $\boldsymbol{g}$, Chemogenetic activation or inhibition of the CLA-PFC pathway did not affect locomotion. Data were presented s mean \pm SEM $n=7 /$ group. ${ }^{*} p<0.05$, compared with saline group. 


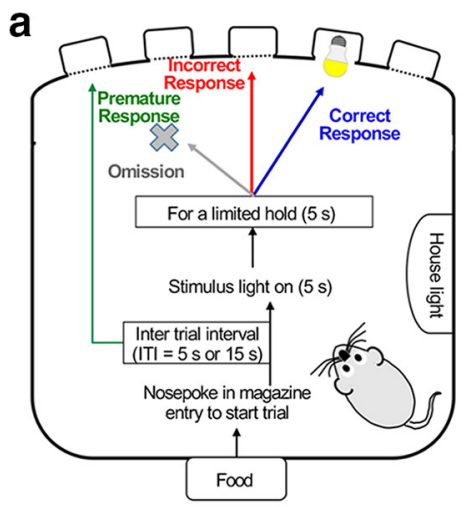

C

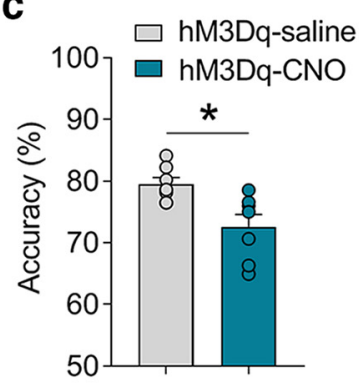

f

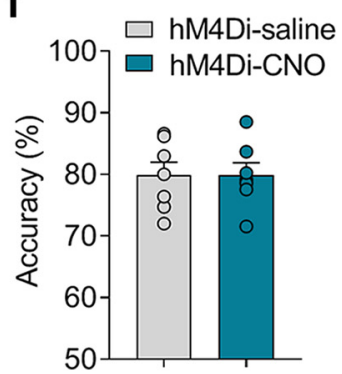

b

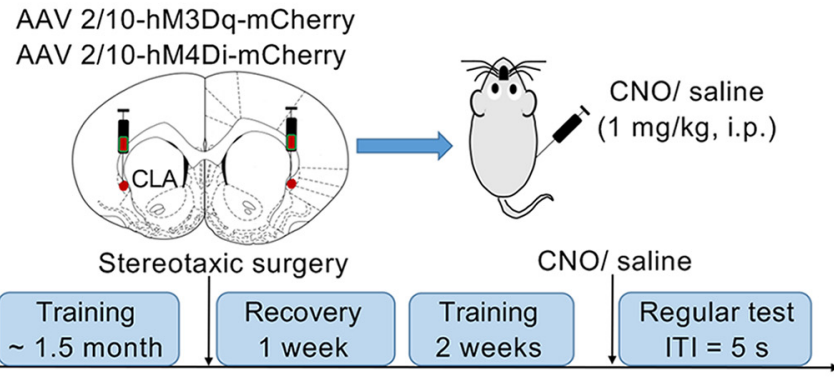

d

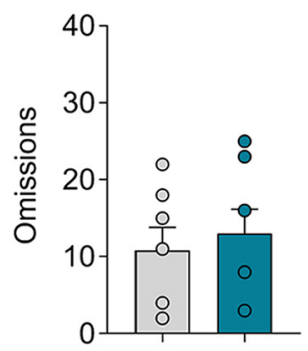

g

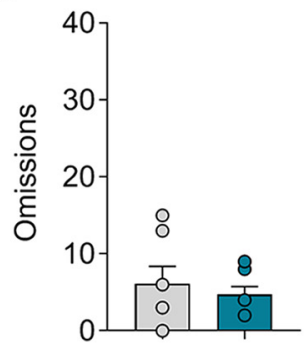

e

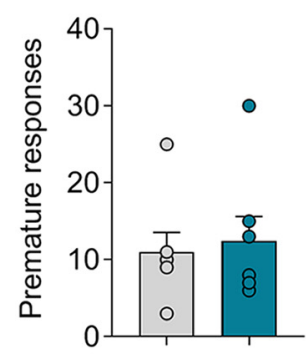

h

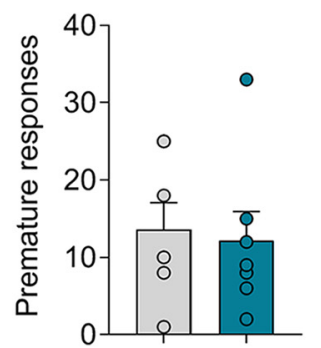

Figure 3. Chemogenetic activation of the claustrum disrupts attention. $\boldsymbol{a}$, The process of the 5-CSRTT task. Three parameters including accuracy, omission, and premature response were analyzed. $\boldsymbol{b}$, Experimental timeline and a diagram showing chemogenetic activation of the CLA ( $n=7 /$ group). $\boldsymbol{c}-\boldsymbol{e}$, In the regular test, chemogenetic activation of the CLA reduced accuracy but did not affect omissions or premature responses. $\boldsymbol{f}-\boldsymbol{h}$, In the regular test, chemogenetic inhibition of the CLA did not affect accuracy, omissions, or premature responses. Data were presented as mean \pm SEM. ${ }^{*} p<0.05$.

these results indicate that the CLA negatively control the activity of the PFC, which is consistent with a recent electrophysiological study reporting an inhibitory control of the PFC by the CLA (Jackson et al., 2018).

Before conducting the behavioral tests in the 5-CSRTT task, we wanted to examine whether the CLA-PFC pathway would induce abnormal motor activity in rats. We microinjected $\mathrm{CNO}$ into the PFC of rats expressing hM3Dq or hM4Di in the CLA. We performed a locomotion test $10 \mathrm{~min}$ after microinjection to let the rats fully recover from the microinjection procedure. We observed that all groups of rats showed similar levels of locomotor activity (two-way repeated-measures ANOVA; hM3Dq: $F_{(5,60)}=0.30, p=0.91$; hM4Di: $F_{(5,60)}=0.43, p=0.82 ; n=$ 7/group), indicating that chemogenetic modulation of the CLAPFC pathway did not produce motor deficits (Fig. $2 g$ ).

\section{Chemogenetic activation of the claustrum disrupts attention}

Given that the CLA projects to a broad range of cortical areas (Atlan et al., 2017; White et al., 2017), we would like first to see whether chemogenetic modulation of the activity of the entire CLA affects the behavioral performances in the 5-CSRTT. We analyzed three major parameters in the 5-CSRTT task, including accuracy (correct responses/ total responses) and omissions to measure attention as well as premature responses to measure impulsivity (Bari et al., 2008; Fig. 3a). All rats were under food restriction by following a standard protocol (Bari et al., 2008), and food (15 g/ d) was provided $1 \mathrm{~h}$ after the training or test session (2:00-4:00 P.M.) each day. After being well trained in the 5 -CSRTT task, rats received microinjections of AAV2/10hM3Dq-mCherry or AAV2/10-hM4Di-mCherry into the CLA. Behavioral tests were conducted 3 weeks after the microinjection. CNO was administered intraperitoneally $(1 \mathrm{mg} / \mathrm{kg})$ to modulate the overall activity of the CLA (Fig. 3b). We found that activation of the CLA significantly attenuated the accuracy (unpaired twotailed $t$ test: $t_{(12)}=3.01, p<0.01$; Fig. $\left.3 c\right)$ but not omissions $\left(t_{(12)}\right.$ $=0.49, p=0.63$; Fig. $3 d)$ or premature responses $\left(t_{(12)}=0.75\right.$, $p=0.73$; Fig. $3 e$ ), suggesting that activation of the CLA induced attention deficit. This result is consistent with recent studies suggesting critical roles of CLA in regulating other attention-related tasks (Atlan et al., 2018; White et al., 2018). However, inhibition of the overall activity of the CLA by a systemic injection of CNO $(1 \mathrm{mg} / \mathrm{kg}$, i.p.) did not change any behaviors in the 5-CSRTT task (accuracy, $t_{(12)}=0.02, p=0.98$; omissions, $t_{(12)}=0.58, p=0.57$; premature responses, $t_{(12)}=0.28, p=0.78$; Fig. $\left.3 f-h\right)$. These results indicated that the claustrum participates in the regulation of attention. 
a

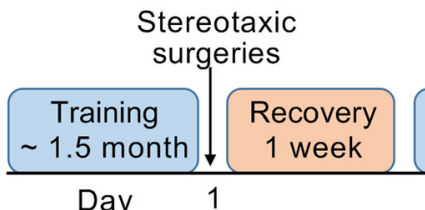

Day

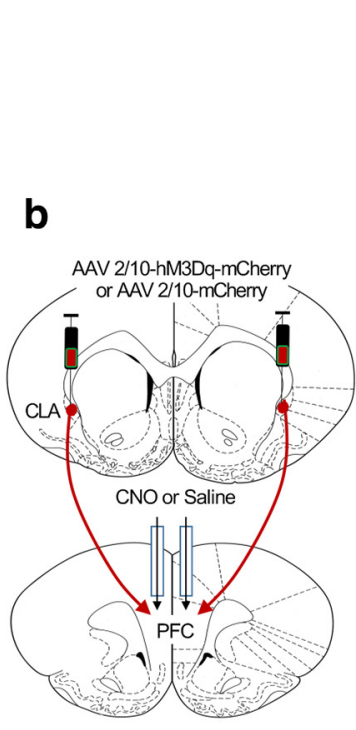

C

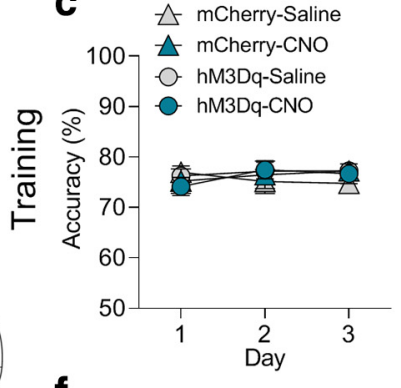

f
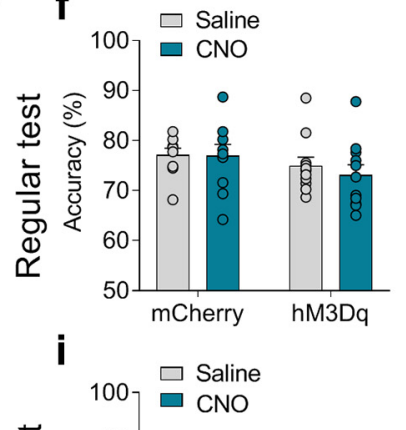

Microinjection

$\left.\begin{array}{c}\text { Training } \\ 2 \text { weeks }\end{array}\right]\left[\begin{array}{c}\text { Regular test } \\ \mathrm{ITI}=5 \mathrm{~s}\end{array}\right]$

23

d

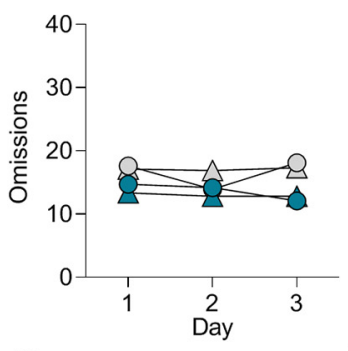

g
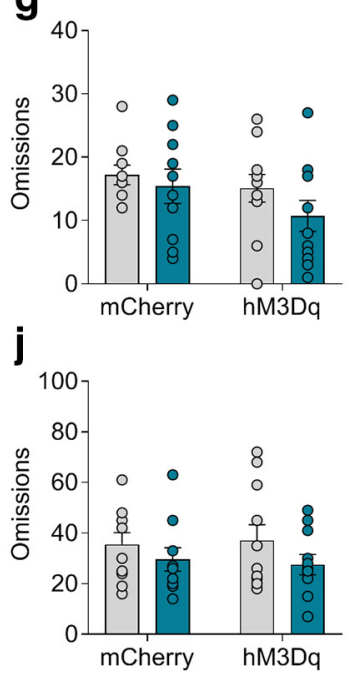

Microinjection of CNO/ Saline

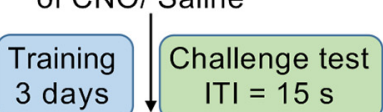

27

e

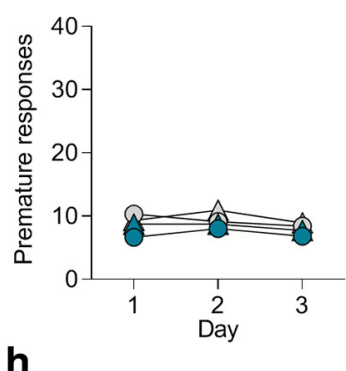

h

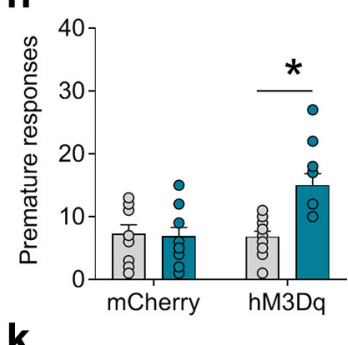

k

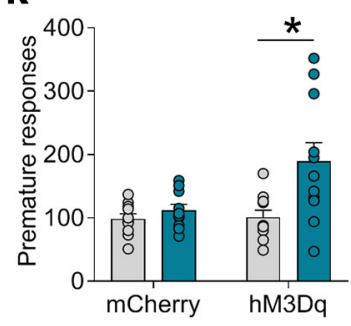

Figure 4. Chemogenetic activation of the CLA-PFC pathway induced impulsive-like behavior in rats. $\boldsymbol{a}$, Experimental timeline: four groups of rats ( $n=10-11 / \mathrm{group})$ received training in the 5-CSRTT, stereotaxic surgery, the regular test (ITI $=5 \mathrm{~s})$, and the challenge test (ITI = $15 \mathrm{~s})$. $\boldsymbol{b}$, Representative figure showing selective activation of the CLA-PFC pathway by excitatory DREADD hM3Dq. c-e, In the last $3 \mathrm{~d}$ of training, no difference was found in the accuracy, omissions, or premature responses. $\boldsymbol{f}-\boldsymbol{h}$, In the regular test, chemogenetic activation of the CLA-PFC pathway increased premature responses but not accuracy or omissions. $\boldsymbol{i}-\boldsymbol{k}$, Chemogenetic activation of the CLA-PFC pathway increased premature responses without influencing accuracy or omissions in a challenge test. Data were presented as mean \pm SEM. ${ }^{*} p<0.05$.

\section{Chemogenetic activation of the CLA-PFC pathway induces impulsivity}

To examine the role of CLA-PFC pathway in attention and impulsivity, we first tested the effect of activation of the CLA-PFC pathway on the performances of the 5-CSRTT task (Fig. 4a). The experimental design was similar to the CLA-PFC pathway experiment as described above, except that rats were microinjected with $\mathrm{CNO}$ via cannulae into the PFC $10 \mathrm{~min}$ before the tests (Fig. $4 b)$. Three-way ANOVA (treatment $\times$ virus $\times$ time) was conducted to analyze the last $3 \mathrm{~d}$ of training. All groups of rats $(n=$ $10-11$ /group $)$ showed the same levels of accuracy $\left(F_{(2,114)}=0.12\right.$, $p=0.86)$, omissions $\left(F_{(2,114)}=0.22, p=0.80\right)$, or premature responses $\left(F_{(2,114)}=0.49, p=0.61\right.$; Fig. $\left.4 c-e\right)$. Two-way ANOVA (treatment $X$ virus) was separately conducted to analyze the accuracy, omissions, and premature responses in the regular and challenge tests. In a regular test during which the ITI was set as $5 \mathrm{~s}$, we found that chemogenetic activation of the CLA-PFC pathway did not affect accuracy $\left(F_{(1,37)}=0.21, p=0.65\right.$; Fig. $\left.4 f\right)$ or omissions $\left(F_{(1,37)}=0.32, p=0.58\right.$; Fig. $\left.4 g\right)$, but significantly increased premature responses (two-way ANOVA; $F_{(1,37)}=9.14, p=$ 0.004; hM3Dq-saline vs hM3Dq-CNO: $t=4.18, p<0.01$; Fig. $4 h$ ). CNO treatment did not affect the behaviors of rats microin- jected with a control virus expressing mCherry (all $p>0.05$; Fig. $4 i-k)$. To further confirm the role of the CLA-PFC pathway, we performed a challenge test, during which the ITI was increased to 15 s (Amitai and Markou, 2011). The premature responses of control rats were markedly increased in the challenge test (premature responses of the mCherry-saline group; regular test vs challenge test, $t_{(18)}=10.94, p<0.01$; Fig. $\left.4 h, k\right)$. Chemogenetic activation of the CLA-PFC pathway further increased premature responses (two-way ANOVA; $F_{(1,38)}=4.35, p=0.04$; hM3Dqsaline vs hM3Dq-CNO: $t=3.53, p<0.01)$ without influencing accuracy $\left(F_{(1,38)}=1.16, p=0.23\right)$ or omissions $\left(F_{(1,38)}=0.14\right.$, $p=0.72$; Fig. $3 i-k)$ in the challenge test. Together, these results indicate that activation of the CLA-PFC pathway induces impulsivity in rats.

\section{Chemogenetic inhibition of the CLA-PFC pathway reduces impulsivity}

We then asked whether the CLA-PFC pathway would regulate impulsivity bidirectionally. We used AAV2/10-hM4Di-mCherry to selectively inhibit the CLA-PFC pathway. The experimental time course was similar to what was described in the above CLAPFC hM3Dq experiment (Fig. 4a,b), except that hM4Di virus 

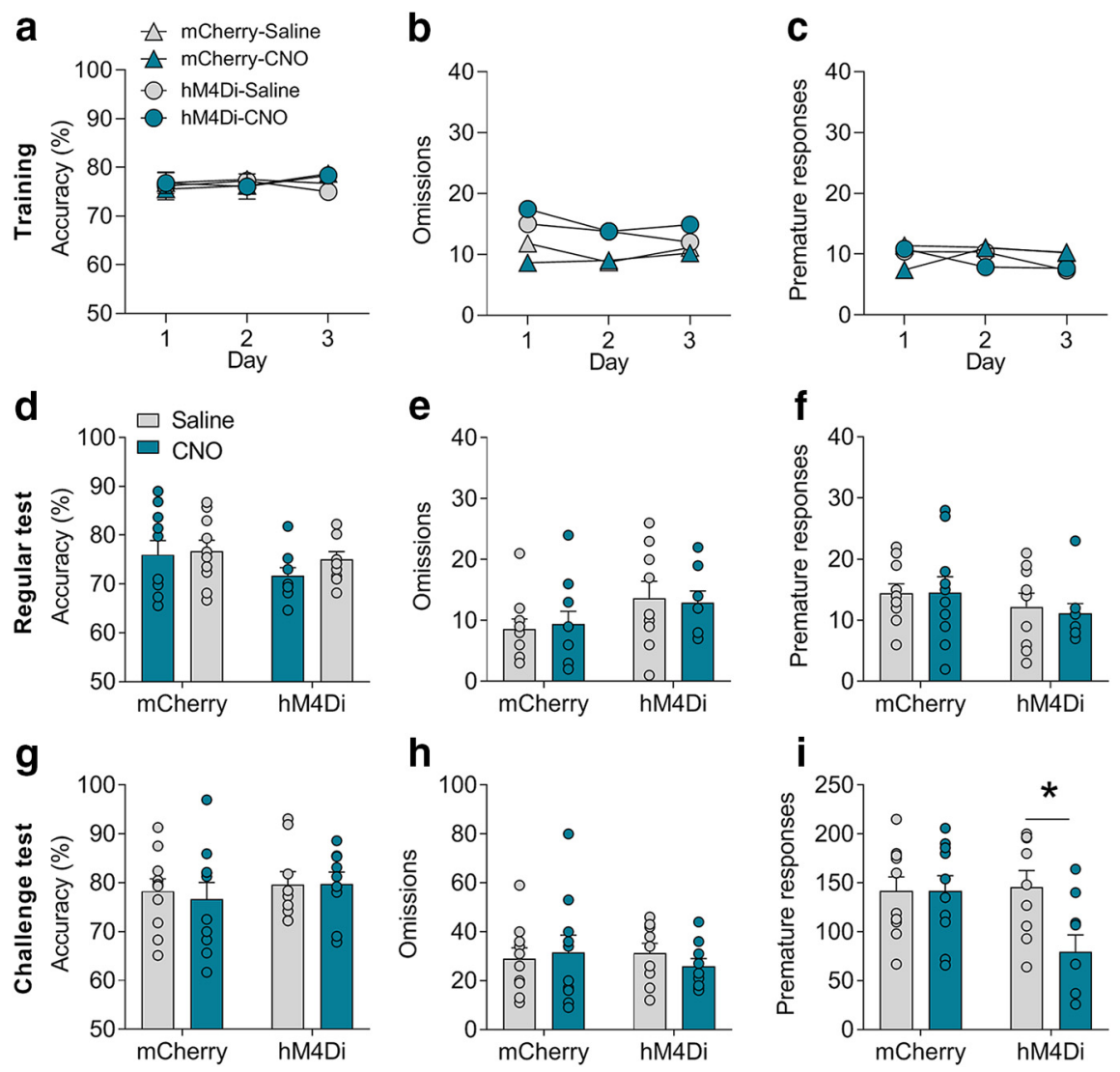

Figure 5. Chemogenetic inhibition of the CLA-PFC pathway attenuated impulsive-like behavior in rats. $\boldsymbol{a}-\boldsymbol{c}$, In the last $3 \mathrm{~d}$ of training, no difference was found in the accuracy, omissions, or premature responses between two groups ( $n=9-10 /$ group). $\boldsymbol{d}-\boldsymbol{f}$, In the regular test, chemogenetic inhibition of the CLA-PFC pathway did not affect accuracy, omissions, or premature responses. $\boldsymbol{g}-\boldsymbol{i}$, In the challenge test, chemogenetic inhibition of the CLA-PFC pathway decreased premature responses but did not affect accuracy or omissions. Data were presented as mean \pm SEM. ${ }^{*} p<0.05$.

rather than hM3Dq virus was administered into the CLA. Threeway ANOVA (treatment $\times$ virus $\times$ time) was conducted to analyze the behavioral performance in the last $3 \mathrm{~d}$ of training, indicating no significant difference in accuracy $\left(F_{(2,102)}=0.04\right.$, $p=0.95)$, omissions $\left(F_{(2,102)}=0.45, p=0.64\right)$, or premature responses $\left(F_{(2,102)}=0.74, p=0.48\right.$; Fig. $\left.5 a-c\right)$. Two-way ANOVA (treatment $X$ virus) was separately conducted to analyze the accuracy, omissions, and premature responses in the regular and challenge tests. However, we did not observe any significant differences in the accuracy $\left(F_{(1,34)}=0.35, p=0.56\right)$, omissions $\left(F_{(1,34)}=0.12, p=0.71\right)$, or premature responses $\left(F_{(1,34)}=0.08\right.$, $p=0.77)$ among the groups of rats in the regular test (ITI $=5 \mathrm{~s}$ ) after inhibition of the CLA-PFC pathway, indicating that inhibition of the CLA-PFC pathway did not affect behavioral performance in the regular test (Fig. $5 d-f$ ). We suspected that this might be because of a floor effect given that the premature responses were relatively low in the regular test. Interestingly, we found a significant effect on the premature responses $\left(F_{(1,34)}=\right.$ $4.23, p=0.047)$ but not in the accuracy $\left(F_{(1,34)}=0.10, p=0.76\right)$ or omissions $\left(F_{(1,34)}=0.63, p=0.43\right)$ in the challenge test (ITI $=$ $15 \mathrm{~s}$; Fig. $5 g-i)$. Post hoc analysis revealed that treatment of CNO reduced premature responses in the hM4Di rats $\left(t_{(34)}=2.83, p=\right.$ $0.02)$ but not in the mCherry rats $\left(t_{(34)}=0.00, p>0.99\right)$, indicating that inhibition of the CLA-PFC pathway significantly reduced the premature responses. These results indicated that inhibition of the CLA-PFC pathway reduced impulsivity.

\section{The CLA-PFC pathway regulates METH-induced impulsivity} Impulsivity plays a critical role in regulating several mental illnesses such as drug addiction (Goldstein and Volkow, 2011). For example, in our previous study, we have shown that acute treatment with $\mathrm{METH}$ increased premature responses in the 5-CSRTT task (Siemian et al., 2017). To determine whether the CLA-PFC pathway would affect impulsivity induced by a drug of abuse, we tested the effects of inhibition of the CLA-PFC pathway on METH-induced impulsivity in the 5-CSRTT task (Fig. 6a). Three-way ANOVA (treatment $\times$ virus $\times$ METH doses) was conducted to analyze the accuracy, omissions, and premature responses. We found that $\mathrm{METH}$ dosedependently increased premature responses (main effect of METH: $F_{(2,102)}=$ 35.56, $p<0.0001$ ) and omissions (main effect of METH: $F_{(2,102)}=43.90, p<$ $0.0001)$. Chemogenetic inhibition of the CLA-PFC pathway reduced $1 \mathrm{mg} / \mathrm{kg}$ $\mathrm{METH}$-induced premature responses (main effect of CNO treatment: $F_{(1,102)}=$ $6.60, p=0.01$; hM4Di-saline vs hM4Di$\mathrm{CNO}$ at $1 \mathrm{mg} / \mathrm{kg} \mathrm{METH}, t_{(102)}=3.59, p=$ 0.03 ) but not omissions (main effect of CNO treatment: $F_{(1,102)}=0.02, p=0.90$; Fig. $6 b-d)$. The core property of psychostimulants is that this kind of drugs induces hyperactivity after acute administration. We then tested whether the CLAPFC pathway regulates METH-induced hyperactivity (Siemian et al., 2017). However, we found that chemogenetic inhibition of the CLA-PFC had no effect on METH-induced hyperactivity in a locomotor test. Two-way ANOVA analysis (CNO treatment $\times$ METH) showed a main effect of METH $\left(F_{(1,20)}=32.74, p<0.001\right)$, but not main effect of CNO treatment $\left(F_{(1,20)}=0.02, p=0.80\right)$ or interaction of METH $\times$ CNO treatment $\left(F_{(1,20)}=0.04, p=0.85\right.$; Fig. $\left.6 f\right)$ These results suggest that inhibition of the CLA-PFC pathway selectively attenuates the effect of METH on impulsivity.

\section{Discussion}

The CLA connects with most parts of the neocortex (Atlan et al., 2017). Unlike the thalamic nuclei which predominately innervate layers II/III and IV of cortices (Herkenham, 1980), CLA inputs primarily target deeper layers such as IV, V, and VI (LeVay and Sherk, 1981). The particular anatomical characteristics of the CLA make it a theoretically ideal brain region for processing in the context of sensation and perception (Goll et al., 2015). Interestingly, a clinical report showed that stimulation of the CLA resulted in a disruption of consciousness, e.g., a complete arrest of volitional behavior, unresponsiveness, and amnesia, suggesting that the CLA may be involved in maintaining consciousness (Koubeissi et al., 2014). However, no further similar clinical or preclinical study has been reported. In addition to, because the CLA is a key note connecting a broad range of brain regions, it is hypothesized that the CLA may integrate or coordinate cortical information (Smith and Alloway, 2010; Smith et al., 2012). Al- 
though there are many hypotheses of the fundamental role of the CLA, recent studies predominantly support the hypothesis that the CLA may regulate attention (Goll et al., 2015). The CLA may control the output of the cortical representations to determine which sensory modality to be attended (Goll et al., 2015). This was indirectly tested by a recent study showing that the CLA controls the ability of resilience to sensory distractions. By using the automated two-alternative forced-choice task and a naturalistic task of maternal pup retrieval, it was demonstrated that inhibition of the CLA disrupted the behavioral performances upon introduction of a distractor, indicating a critical role of the CLA in supporting resilience to a distraction (Atlan et al., 2018). The CLA may also serve as a neural center of top-down control from the frontal cortex to modulate the sensory and motor cortical modalities. A study showed that the CLA receives rich innervation from the anterior cingulate cortex (ACC) and that optogenetic inhibition of the neural projections from the ACC to the CLA (ACC-CLA pathway) disrupted percentage correct responses in the 5-CSRTT, suggesting a critical role of CLA in regulating the top-down action control (White et al., 2018). Also, the CLA may interact with the limbic regions such as hippocampus and amygdala to transmit salient information and regulate state-dependent attention (Goll et al., 2015). For example, the entorhinal cortexprojecting neurons in CLA could be activated by novel context and participated in the formation of hippocampus-dependent contextual fear memory (Kitanishi and Matsuo, 2017).

The role of the CLA in regulating attention could be explained by direct regulation of the CLA on sensory and motor cortexes. Electrical stimulation of the CLA attenuated the spontaneous activity and firing properties of visual cortical neurons in the cats (Ptito and Lassonde, 1981). Stimulation of the CLA also reduced spontaneous activity of pyramidal tract neurons in the motor cortex of cats (Crescimanno et al., 1984). Although it is unclear how the electrical stimulation would activate the CLA because both excitatory pyramidal neurons and inhibitory interneurons are richly expressed in the CLA, these results may imply an inhibitory effect of the CLA on sensory and motor cortexes. If the CLA negatively controls sensory and motor cortexes, activation of the CLA would inhibit the activity of these cortexes, which presumably disrupts attention. Consistent with this, our results showed that chemogenetic activation of the CLA disrupted percentage correct responses in the 5-CSRTT, indicating that the CLA negatively controls attention. These results seem to be inconsistent with the results that inhibition of the ACC-CLA pathway and inhibition of the CLA disrupted attention (Atlan et al., 2018; White et al., 2018). One explanation is that whereas activation of the CLA may inhibit the activity of the downstream cortexes, inhibition of the CLA may overactivate the cortical areas to pre- vent the brain from processing new information. Accordingly, it might be necessary that the CLA needs to be activated at an appropriate level to maintain a regular attention.

A recent study demonstrated that the CLA negatively controls the PFC (Jackson et al., 2018). By analyzing the expression level of c-Fos, a marker of neural activation, our results indicated that chemogenetic activation and inhibition of the CLA-PFC pathway attenuated and increased the overall activity of the PFC. The PFC has been greatly implicated in regulating impulsivity (Bari and Robbins, 2013). Studies showed that pharmacological lesion or inhibition of the glutamatergic system of the PFC-induced impulsivity (Chudasama et al., 2003; Paine et al., 2011). Consistently, our results indicated that the CLA-PFC pathway negatively regulates impulsivity, e.g., producing impulsivity by activation of the pathway and attenuating impulsivity by inhibition of the pathway. Intriguingly, modulation of the CLA-PFC pathway did not affect behavioral performances of attention in the 5-CSRTT. It seems paradoxical that the CLA regulates attention while the CLA-PFC pathway does not. However, because the CLA extensively innervates the neocortex, it is not surprising that modulating the activity of the CLA and the activity of the particular CLA-PFC pathway affects attention and impulsivity in a different way. Evidence also indicates that the infralimbic cortex 
(the ventral part of the medial PFC), rather than the more dorsal prelimbic cortex is associated with impulsivity (Chudasama et al., 2003; Murphy et al., 2005). However, because this is the first study to investigate the CLA-PFC pathway in the 5-CSRTT, we did not distinguish the dorsal and ventral parts of the medial PFC in our study. It will be of great interest to determine the precise neural circuitry in future studies. Impulsivity is a broad concept which includes several aspects including reflection impulsivity, impulsive action, and impulsive choice (Wakabayashi et al., 2019). The premature response in the 5-CSRTT is primarily used to evaluate impulsive action (Dalley et al., 2011). Moreover, the performance of animals in the 5-CSRTT was maintained by food self-administration and required a specific set of function in regulating motivation, perception, and rewarding, which potentially interacts with impulsivity. Future studies are required to address how exactly the CLA-PFC pathway regulates impulsivity. It is also noted that only male rats were used in this study. Given that there exists sex differences on the performance in the 5-CSRTT, the role of the CLA-PFC pathway in regulating impulsivity might not generalize to female animals (Bayless et al., 2012; Weafer and de Wit, 2014; Grissom and Reyes, 2019).

Furthermore, we demonstrated that chemogenetic inhibition of the CLA-PFC pathway attenuated METH-induced impulsivity without affecting $\mathrm{METH}$-induced attention deficit or hyperactivity, indicating that the CLA-PFC pathway selectively controls impulsivity induced by METH. Drug-induced impulsivity plays a role in the development of drug addiction, and high impulsivity is associated with compulsive drug use (Dalley et al., 2011; Goldstein and Volkow, 2011). Except for compulsive drug use, drug addiction is also characterized by a high rate of relapse (Dong et al., 2017). The PFC was extensively implicated in the extinction of drug-seeking and relapse-like behaviors such as drug-paired cues, and drug-induced reinstatements of drugseeking (Kalivas and Volkow, 2005). Because the CLA directly controls the activity of PFC (Jackson et al., 2018), it is possible that the CLA may participate in the regulation of drug relapse. Thus, controlling impulsivity by modulating the CLA-PFC pathway might be a strategy to combat drug addiction and relapse.

In summary, we identified the CLA as a brain region that controls the activity of the PFC and revealed a critical role of the CLA-PFC pathway in regulating impulsivity. Our study may shed light on the understanding of impulsivity-related disorders such as drug addiction and attention-deficit/hyperactivity disorder (Nigg, 2003).

\section{References}

Amitai N, Markou A (2011) Comparative effects of different test day challenges on performance in the 5-choice serial reaction time task. Behav Neurosci 125:764-774.

Atlan G, Terem A, Peretz-Rivlin N, Groysman M, Citri A (2017) Mapping synaptic cortico-claustral connectivity in the mouse. J Comp Neurol 525: 1381-1402.

Atlan G, Terem A, Peretz-Rivlin N, Sehrawat K, Gonzales BJ, Pozner G, Tasaka GI, Goll Y, Refaeli R, Zviran O, Lim BK, Groysman M, Goshen I, Mizrahi A, Nelken I, Citri A (2018) The claustrum supports resilience to distraction. Curr Biol 28:2752-2762.e7.

Bari A, Robbins TW (2013) Inhibition and impulsivity: behavioral and neural basis of response control. Prog Neurobiol 108:44-79.

Bari A, Dalley JW, Robbins TW (2008) The application of the 5-choice serial reaction time task for the assessment of visual attentional processes and impulse control in rats. Nat Protoc 3:759-767.

Bayless DW, Darling JS, Stout WJ, Daniel JM (2012) Sex differences in attentional processes in adult rats as measured by performance on the 5-choice serial reaction time task. Behav Brain Res 235:48-54.

Brown SP, Mathur BN, Olsen SR, Luppi PH, Bickford ME, Citri A (2017)
New breakthroughs in understanding the role of functional interactions between the neocortex and the claustrum. J Neurosci 37:10877-10881.

Chudasama Y, Passetti F, Rhodes SE, Lopian D, Desai A, Robbins TW (2003) Dissociable aspects of performance on the 5-choice serial reaction time task following lesions of the dorsal anterior cingulate, infralimbic and orbitofrontal cortex in the rat: differential effects on selectivity, impulsivity and compulsivity. Behav Brain Res 146:105-119.

Crescimanno G, Salerno MT, Cortimiglia R, Amato G, Infantellina F (1984) Functional relationship between claustrum and pyramidal tract neurons, in the cat. Neurosci Lett 44:125-129.

Crick FC, Koch C (2005) What is the function of the claustrum? Philos Trans R Soc Lond B Biol Sci 360:1271-1279.

Dalley JW, Everitt BJ, Robbins TW (2011) Impulsivity, compulsivity, and top-down cognitive control. Neuron 69:680-694.

Dong Y, Taylor JR, Wolf ME, Shaham Y (2017) Circuit and synaptic plasticity mechanisms of drug relapse. J Neurosci 37:10867-10876.

Goldstein RZ, Volkow ND (2011) Dysfunction of the prefrontal cortex in addiction: neuroimaging findings and clinical implications. Nat Rev Neurosci 12:652-669.

Goll Y, Atlan G, Citri A (2015) Attention: the claustrum. Trends Neurosci 38:486-495.

Grissom NM, Reyes TM (2019) Let's call the whole thing off: evaluating gender and sex differences in executive function. Neuropsychopharmacology 44:86-96.

Herkenham M (1980) Laminar organization of thalamic projections to the rat neocortex. Science 207:532-535.

Jackson J, Karnani MM, Zemelman BV, Burdakov D, Lee AK (2018) Inhibitory control of prefrontal cortex by the claustrum. Neuron 99:10291039.e4.

Kalivas PW, Volkow ND (2005) The neural basis of addiction: a pathology of motivation and choice. Am J Psychiatry 162:1403-1413.

Kim J, Matney CJ, Roth RH, Brown SP (2016) Synaptic organization of the neuronal circuits of the claustrum. J Neurosci 36:773-784.

Kim S, Lee D (2011) Prefrontal cortex and impulsive decision making. Biol Psychiatry 69:1140-1146.

Kitanishi T, Matsuo N (2017) Organization of the claustrum-to-entorhinal cortical connection in mice. J Neurosci 37:269-280.

Koubeissi MZ, Bartolomei F, Beltagy A, Picard F (2014) Electrical stimulation of a small brain area reversibly disrupts consciousness. Epilepsy Behav 37:32-35.

LeVay S, Sherk H (1981) The visual claustrum of the cat: I. Structure and connections. J Neurosci 1:956-980.

Liu JF, Siemian JN, Seaman R Jr, Zhang Y, Li JX (2017) Role of TAAR1 within the subregions of the mesocorticolimbic dopaminergic system in cocaine-seeking behavior. J Neurosci 37:882-892.

Liu JF, Seaman R Jr, Siemian JN, Bhimani R, Johnson B, Zhang Y, Zhu Q, Hoener MC, Park J, Dietz DM, Li JX (2018) Role of trace amineassociated receptor 1 in nicotine's behavioral and neurochemical effects. Neuropsychopharmacology 43:2435-2444.

Miller EK (2000) The prefrontal cortex and cognitive control. Nat Rev Neurosci 1:59-65.

Murphy ER, Dalley JW, Robbins TW (2005) Local glutamate receptor antagonism in the rat prefrontal cortex disrupts response inhibition in a visuospatial attentional task. Psychopharmacology 179:99-107.

Nigg JT (2003) Response inhibition and disruptive behaviors: toward a multiprocess conception of etiological heterogeneity for ADHD combined type and conduct disorder early-onset type. Ann N Y Acad Sci 1008:170-182.

Paine TA, Slipp LE, Carlezon WA Jr (2011) Schizophrenia-like attentional deficits following blockade of prefrontal cortex $\mathrm{GABA}_{\mathrm{A}}$ receptors. Neuropsychopharmacology 36:1703-1713.

Ptito M, Lassonde MC (1981) Effects of claustral stimulation on the properties of visual cortex neurons in the cat. Exp Neurol 73:315-320.

Robbins TW (2002) The 5-choice serial reaction time task: behavioural pharmacology and functional neurochemistry. Psychopharmacology 163:362-380.

Roth BL (2016) DREADDs for neuroscientists. Neuron 89:683-694.

Siemian JN, Xue Z, Blough BE, Li JX (2017) Comparison of some behavioral effects of D- and L-methamphetamine in adult male rats. Psychopharmacology 234:2167-2176.

Smith JB, Alloway KD (2010) Functional specificity of claustrum connec- 
tions in the rat: interhemispheric communication between specific parts of motor cortex. J Neurosci 30:16832-16844.

Smith JB, Radhakrishnan H, Alloway KD (2012) Rat claustrum coordinates but does not integrate somatosensory and motor cortical information. J Neurosci 32:8583-8588.

Wakabayashi KT, Feja M, Baindur AN, Bruno MJ, Bhimani RV, Park J, Hausknecht K, Shen RY, Haj-Dahmane S, Bass CE (2019) Chemogenetic activation of ventral tegmental area GABA neurons, but not mesoaccumbal GABA terminals, disrupts responding to reward-predictive cues. Neuropsychopharmacology 44:372-380.

Weafer J, de Wit H (2014) Sex differences in impulsive action and impulsive choice. Addict Behav 39:1573-1579.

White MG, Cody PA, Bubser M, Wang HD, Deutch AY, Mathur BN (2017)
Cortical hierarchy governs rat claustrocortical circuit organization. The J Comp Neurol 525:1347-1362.

White MG, Panicker M, Mu C, Carter AM, Roberts BM, Dharmasri PA, Mathur BN (2018) Anterior cingulate cortex input to the claustrum is required for top-down action control. Cell Rep 22:84-95.

Xue Z, Siemian JN, Johnson BN, Zhang Y, Li JX (2018) Methamphetamineinduced impulsivity during chronic methamphetamine treatment in rats: effects of the TAAR 1 agonist RO5263397. Neuropharmacology 129: $36-46$.

Yan W, Liu JF, Han Y, Zhang W, Luo YX, Xue YX, Zhu WL, Yang C, Chen WH, Guo HL, Ma YN, Yuan K, Wang JS, Shi J, Lu L (2018) Protein kinase $\mathrm{m} \zeta$ in medial prefrontal cortex mediates depressive-like behavior and antidepressant response. Mol Psychiatry 23:1878-1891. 